\title{
Energy-Harvesting in Cooperative AF Relaying Networks Over Log-Normal Fading Channels
}

\author{
Khaled M. Rabie*, Abdelhamid Salem*, Emad Alsusa* and Mohamed-Slim Alouini ${ }^{\ddagger}$ \\ *School of Electrical \& Electronic Engineering, The University of Manchester, Manchester, UK, M13 9PL \\ ${ }^{\ddagger}$ King Abdullah University of Science and Technology (KAUST), Thuwal, Mekkah Province, Saudi Arabia \\ Email: \{khaled.rabie, abdelhamid.salem, e.alsusa\}@manchester.ac.uk; slim.alouini@kaust.edu.sa
}

\begin{abstract}
Energy-harvesting (EH) and wireless power transfer are increasingly becoming a promising source of power in future wireless networks and have recently attracted a considerable amount of research, particularly on cooperative two-hop relay networks in Rayleigh fading channels. In contrast, this paper investigates the performance of wireless power transfer based two-hop cooperative relaying systems in indoor channels characterized by log-normal fading. Specifically, two EH protocols are considered here, namely, time switching relaying (TSR) and power splitting relaying (PSR). Our findings include accurate analytical expressions for the ergodic capacity and ergodic outage probability for the two aforementioned protocols. Monte Carlo simulations are used throughout to confirm the accuracy of our analysis. The results show that increasing the channel variance will always provide better ergodic capacity performance. It is also shown that a good selection of the EH time in the TSR protocol, and the power splitting factor in the PTS protocol, is the key to achieve the best system performance.
\end{abstract}

Index Terms-AF relaying, energy-harvesting (EH), ergodic capacity, log-normal fading, wireless power transfer.

\section{INTRODUCTION}

$\mathbf{P}$ ROLONGING the lifetime of energy-constrained wireless networks has always been a central point of research. This is even more appealing in low-power consumption wireless devices where replacing or recharging their batteries is undesirable. In such scenarios, a more convenient solution is to make the wireless network power-independent by simply scavenging energy from the surroundings, such as electromagnetic waves. The advent of using electromagnetic waves to concurrently carry information and energy signals, also known as simultaneous wireless information and power transfer (SWIPT), has drawn significant research interest in recent years [1], [2]. In particular, a considerable amount of this research has focused on amplify-and-forward (AF) and decodeand-forward (DF) cooperative communication networks with energy-harvesting (EH) relays. For example, the authors in [3] analyzed the performance of AF EH relaying for halfduplex systems where a greedy switching policy based on time-switching is introduced. In [4], the authors evaluated the performance of a one-way AF relaying network in terms of the system throughput. This study proposed two relaying protocols based on power splitting relaying (PSR) and time switching relaying (TSR). In the former protocol, the received signal power is split into two parts, one for information processing and the other for $\mathrm{EH}$, whereas in the latter this is achieved by means of time switching between the information receiver and the energy-harvester. In addition, [5] evaluated the performance of a two-way EH relay network in terms of the outage probability and ergodic capacity. Furthermore, the authors in [6] and [7] considered DF relaying with EH constraints. To achieve a better trade-off between information transmission and $\mathrm{EH}$, relay selection in multiple-relay networks were also examined. In this regard, many relay selection techniques are investigated such as time-sharing selection, weighted different selection and threshold-checking selection [8].

All these studies have been limited to Rayleigh fading channels which is used to model the outdoor wireless channel. However, to the best of the authors' knowledge, no work has considered the analysis of $\mathrm{EH}$ and wireless power transfer systems over log-normal fading channels which statistically characterize fading in various radio propagation environments such as indoor and ultra wide-band channels, as well as shadowing effects in outdoor scenarios [9], [10]. This paper is therefore devoted to analyzing the performance of cooperative EH relaying systems over log-normal fading channels. Two well-known EH protocols are considered in this investigation, namely, TSR and PSR. The performance of these schemes is evaluated in terms of the ergodic capacity and the corresponding ergodic outage probability. The resulting accurate analytical expressions provide insights into the behavior of such protocols under different system settings. Monte Carlo simulations are also provided to validate our analysis. Results reveal that the two systems under consideration will always have better performance as the log-normal channel variance increases and that optimizing the EH time in the TSR protocol, and power splitting factor in the PTS protocol, will maximize the achievable ergodic capacity.

The rest of this paper is organized as follows. Section II describes the system model and defines the system performance metrics adopted in this study. Accurate analytical expressions are derived for the ergodic capacity and the ergodic outage probability for the TSR and PSR protocols over the log-normal fading channel in Sections III and IV, respectively. Numerical and simulation examples are presented and discussed in Section V. Finally, Section VI summarizes the paper and outlines the main conclusions.

\section{System Model}

The system model under consideration is shown in Fig. 1. This system consists of a source node transmitting its data to a destination node through an energy-constrained AF relay node. The relay is an EH node which relies entirely on harvesting 


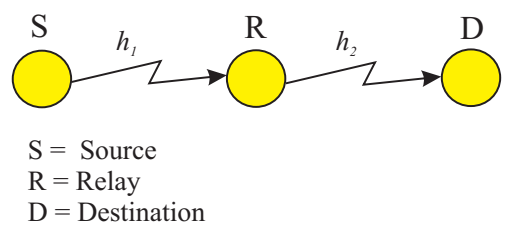

Figure 1: Basic system model with cooperative AF relaying over a lognormal fading channel.

the energy transmitted by the source. The source-to-relay (S-to$\mathrm{R})$ and relay-to-destination (R-to-D) channel gains are denoted as $h_{1}$ and $h_{2}$ and the corresponding distances are $d_{1}$ and $d_{2}$, respectively, see Fig. 1. The two links experience quasi-static block fading; that is, the channels remain constant over the block time, $T$, and vary independently and identically from one block to another according to log-normal distribution with the following probability density function (PDF)

$$
f_{Z}\left(z_{i}\right)=\frac{\xi}{\sqrt{2 \pi} \sigma z_{i}} \exp \left[-\frac{\left(10 \log _{10}\left(z_{i}\right)-\mu\right)^{2}}{2 \sigma^{2}}\right]
$$

where $z_{i}=h_{i}^{2}, i \epsilon\{1,2\}, \xi=10 / \ln (10)$ is a scaling constant, $\mu$ and $\sigma^{2}$ (both in decibels) are the mean and the standard deviation of $10 \log _{10}(h)$, respectively. The corresponding momentgenerating function (MGF) of the PDF in (1), which will be used later in our analysis to derive the ergodic capacity for the various harvesting systems under consideration, is calculated simply as [11, eq. (2.54)]

$$
\begin{aligned}
\Psi_{Z}(s) & =\int_{0}^{\infty} \exp (-s z) f(z) \mathrm{d} z \\
= & \int_{0}^{\infty} \frac{\exp (-s z) \xi}{\sqrt{2 \pi} \sigma z} \exp \left[-\frac{\left(10 \log _{10}(z)-\mu\right)^{2}}{2 \sigma^{2}}\right] \mathrm{d} z
\end{aligned}
$$

No closed-form expression for this MGF is available in the literature. However, it can be expressed, for real $s$, by a series expansion based on Gauss-Hermite integration as [12]

$$
\Psi(s) \triangleq \sum_{n=1}^{N} \frac{w_{n}}{\sqrt{\pi}} \exp \left[-s \exp \left(\frac{\sqrt{2} \sigma x_{n}+\mu}{\xi}\right)\right],
$$

where $N$ denotes the Hermite integration order, $\left\{w_{n}\right\}_{n=1}^{N}$ and $\left\{x_{n}\right\}_{n=1}^{N}$ are the weights and abscissas, respectively. In this paper we will use $N=25$ and the corresponding weights and abscissas are tabulated in [13, Table 25.10].

In our system model, it is assumed that there is no direct link between the source and destination nodes, that is all communications are accomplished over two phases through the relay. In the first phase, the source transmits the energy signal which is harvested by the energy-harvester at the relay, and in the second phase the relay uses this energy to amplify and forward the source signal to the intended destination. Without loss of generality, we also assume that the relay has perfect knowledge of both the S-to-R and R-to-D channels state information as well as the corresponding distances, which are common assumptions in analyzing cooperative communication systems [14]-[16].

\section{A. Ergodic Capacity}

The ergodic capacity is determined as

$$
\mathbb{E}\left[C_{D}\right]=\frac{1}{2} \mathbb{E}\left[\log _{2}\left(1+\gamma_{D}\right)\right],
$$

where $\mathbb{E}[$.$] is the expectation operator, \gamma_{D}$ and $C_{D}$ denote the signal-to-noise ratio (SNR) and the capacity at the destination node, respectively, and the factor $\frac{1}{2}$ is due to the required two time slots for S-to-D transmission.

\section{B. Ergodic Outage Probability}

The ergodic outage probability is an important quality of service measure and is defined as the probability that the ergodic capacity drops below a predetermined threshold, $C_{t h}$. It is given as

$$
P_{\text {out }}=\operatorname{Pr}\left[C_{D}<C_{t h}\right] .
$$

To assess the performance of the system considered here we need to derive analytical expressions for the ergodic capacity and ergodic outage probability with the two EH based systems over the log-normal fading channel.

\section{TIME SWITCHING RELAYING}

The principle of this protocol is illustrated in Fig. 2. As can be seen from Fig. 2a, the time required to transmit one block from the source to the destination is given by $T$ and the EH time during which the relay harvests energy is denoted as $\alpha T$ where $0 \leq \alpha \leq 1$. The remaining time is divided into two slots each of length $(1-\alpha) T / 2$ used for data transmission during phases I and II, respectively. To elaborate, the received signal at the relay in the first phase can be expressed as [17]

$$
y_{r}(t)=\frac{1}{\sqrt{d_{1}^{m}}} \sqrt{P_{s}} h_{1} s(t)+n_{a}(t),
$$

where $m$ is the path loss exponent, $P_{s}$ is the transmitted source power, $s(t)$ is the information signal, $\mathbb{E}\left[|s(t)|^{2}\right]=1$, and $n_{a}(t)$ is the noise at the relay with variance $\sigma_{a}^{2}$. Hence, the harvested energy at the relay during the time period $\alpha T$ can be expressed as

$$
E_{H}=\eta \alpha T\left(\frac{P_{s}}{d_{1}^{m}} h_{1}^{2}+\sigma_{a}^{2}\right),
$$

where $0<\eta<1$ is the EH efficiency determined mainly by the circuitry. In the second phase, the transmitted signal at the relay after the base-band processing and amplification can be given by

$$
r(t)=\frac{1}{\sqrt{d_{1}^{m}}} \sqrt{P_{s} P_{r}} G h_{1} s(t)+\sqrt{P_{r}} G n_{r}(t)
$$

while $P_{r}$ is the relay transmit power, $n_{r}(t)=n_{a}(t)+n_{c}(t)$ where $n_{c}(t)$ is the noise added by the information receiver and $G$ is the relay gain which is given by $G=\frac{1}{\sqrt{\frac{P_{s}^{s}}{d_{1}^{2}} h_{1}^{2}+\sigma_{r}^{2}}}$, and $\sigma_{r}^{2}=\sigma_{a}^{2}+\sigma_{c}^{2}$. Now, the received signal at the destination in the second phase can be written as

$$
y_{D}(t)=\frac{1}{\sqrt{d_{1}^{m} d_{2}^{m}}} \sqrt{P_{s} P_{r}} G h_{1} h_{2} s(t)
$$




$$
+\frac{1}{\sqrt{d_{2}^{m}}} \sqrt{P_{r}} G h_{2} n_{r}(t)+n_{d}(t)
$$

where $n_{d}$ is the noise at the destination node with variance $\sigma_{d}^{2}$. Furthermore, the transmitted power at the relay is related to the harvested energy as $P_{r}=\frac{2 E_{H}}{(1-\alpha) T}$, which can also be written, using (8), as

$$
P_{r}=\frac{2 \eta \alpha}{(1-\alpha)}\left(\frac{P_{s}}{d_{1}^{m}} h_{1}^{2}+\sigma_{a}^{2}\right) .
$$

Now, by substituting (11) into (10), and with some algebraic manipulation, we can express the SNR at the destination as

$$
\gamma_{D}=\frac{2 \eta \alpha P_{s} h_{1}^{2} h_{2}^{2}}{2 \eta \alpha h_{2}^{2} d_{1}^{m} \sigma_{r}^{2}+(1-\alpha) d_{1}^{m} d_{2}^{m} \sigma_{d}^{2}} .
$$

\section{A. Ergodic Capacity for the TSR Protocol}

In this section, we derive an expression for the ergodic capacity for the TSR protocol. To begin with, let

$$
\begin{aligned}
& a_{1}=2 \eta \alpha P_{s}, \\
& a_{2}=(1-\alpha) d_{1}^{m} d_{2}^{m} \sigma_{d}^{2}, \\
& a_{3}=2 \eta \alpha d_{1}^{m} \sigma_{r}^{2}, \\
& \mathcal{X}=a_{1} X, \text { and } \\
& \mathcal{Y}=a_{2} \bar{Y},
\end{aligned}
$$

where $X=h_{1}^{2}$ and $\bar{Y}=h_{2}^{-2}$. Given these definitions, the SNR $\gamma_{D}$ in (12) can be re-expressed in a simpler form as follows

$$
\gamma_{D}=\frac{\mathcal{X}}{\mathcal{Y}+a_{3}} .
$$

Hence, using (5) and (14), the ergodic capacity of the TSR based system can also be expressed as

$$
\mathbb{E}\left[C_{D}\right]=\frac{(1-\alpha)}{2} \mathbb{E}\left[\log _{2}\left(1+\frac{\mathcal{X}}{\mathcal{Y}+a_{3}}\right)\right] .
$$

It should be pointed out that the term $(1-\alpha)$ implies that only during this fraction of time, information transmission takes place as the rest of the time is occupied for EH as discussed earlier.

Lemma 1. It is found in [18] that for any $u, v>0$

$$
\mathbb{E}\left[\ln \left(1+\frac{u}{v}\right)\right]=\int_{0}^{\infty} \frac{1}{s}\left(\Psi_{v}(s)-\Psi_{v, u}(s)\right) d s,
$$

where $\Psi_{v}(s)$ denotes the MGF of the random variable $(R V) v$, defined in (2) whereas, when $v$ and $u$ are independent, $\Psi_{v, u}(s)$ is given by $\Psi_{v, u}(s)=\Psi_{v}(s) \Psi_{u}(s)$.

Using the definition in (16), and since $\mathcal{X}$ and $\mathcal{Y}$ are independent, the destination ergodic capacity of the TSR system in (15) can be obtained as

$$
\mathbb{E}\left[C_{D}\right]=\frac{(1-\alpha)}{2 \ln (2)} \int_{0}^{\infty} \frac{1}{s}\left(1-\Psi_{\mathcal{X}}(s)\right) \Psi_{\mathcal{Y}+a_{3}}(s) d s
$$

where $\Psi_{\mathcal{X}}(s)$ and $\Psi_{\mathcal{Y}+a_{3}}(s)$ are the MGFs of the RVs $\mathcal{X}$ and $\mathcal{Y}+a_{3}$ given by $\Psi_{\mathcal{X}}(s)=\Psi_{X}\left(a_{1} s\right)$ and $\Psi_{\mathcal{Y}+a_{3}}(s)=$

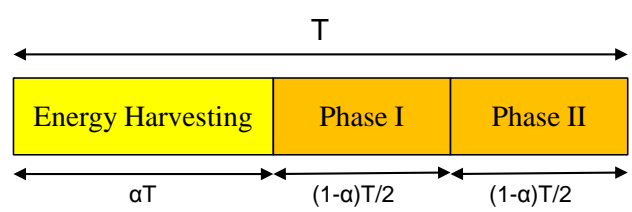

(a) Time frame structure in the TSR protocol.

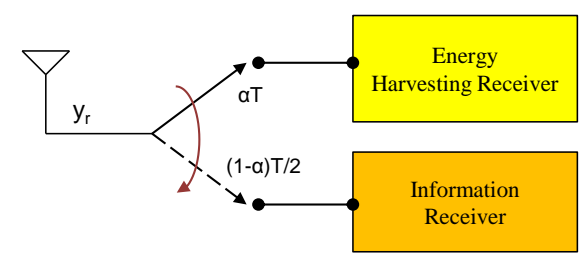

(b) Block diagram of the TSR-based relaying system.

Figure 2: TSR protocol for EH and information signal processing at the relay.

$\Psi_{\bar{Y}}\left(a_{2} s\right) \exp \left(-a_{3} s\right)$, respectively. Since $X$ and $\bar{Y}$ are lognormally distributed with parameters $h_{1}^{2} \sim \mathcal{L N}\left(2 \mu_{h_{1}}, 4 \sigma_{h_{1}}^{2}\right)$ and $h_{2}^{-2} \sim \mathcal{L N}\left(-2 \mu_{h_{2}}, 4 \sigma_{h_{2}}^{2}\right)$, using the series expansion based on Gauss-Hermite integration (4), we can express $\Psi_{\mathcal{X}}(s)$ and $\Psi_{\mathcal{Y}+a_{3}}(s)$ as in (18) and (19), respectively, shown at the top of the next page. Finally, by substituting (18) and (19) into (17), we get the ergodic capacity for the TSR-based system.

\section{B. Ergodic Outage Probability for the TSR Protocol}

To derive the ergodic outage probability for the proposed TSR system, it is more convenient to rewrite (12) as

$$
\gamma_{D}=\frac{a_{1} h_{1}^{2} h_{2}^{-2}}{a_{3} h_{2}^{2}+a_{2}},
$$

where $a_{1}, a_{2}$ and $a_{3}$ are defined in (13). Substituting (20) into (6) yields

$$
\begin{aligned}
P_{\text {out }} & =\operatorname{Pr}\left[\frac{a_{1} X Y}{a_{3} Y+a_{2}}<v\right] \\
& =\operatorname{Pr}\left(Y<\frac{a_{2} \nu}{a_{1} X-a_{3} \nu}\right),
\end{aligned}
$$

where $v=2^{\frac{2 C_{t h}}{1-\alpha}}-1$. The fact that $Y$ is always a positive value implies

$$
P_{\text {out }}= \begin{cases}\operatorname{Pr}\left(Y<\frac{v a_{2}}{a_{1} X-\nu a_{3}}\right), & X<\frac{v a_{3}}{a_{1}} \\ \operatorname{Pr}\left(Y>\frac{v a_{2}}{a_{1} X-v a_{3}}\right)=1, & X>\frac{v a_{3}}{a_{1}} .\end{cases}
$$

With this in mind, the outage probability can now be calculated as in (23), shown at the top of the next page, where $f_{X}($.$) is the PDF of the log-normally distributed \mathrm{RV}, X$, and is given as

$$
f_{X}(z)=\frac{\xi}{z \sqrt{8 \pi \sigma_{h_{1}}^{2}}} \exp \left[-\frac{\left(\xi \ln (z)-2 \mu_{h_{1}}-\xi \ln \left(a_{1}\right)\right)^{2}}{8 \sigma_{h_{1}}^{2}}\right]
$$




$$
\Psi_{\mathcal{X}}(s) \triangleq \sum_{n=1}^{N} \frac{w_{n}}{\sqrt{\pi}} \exp [\underbrace{-2 \eta \alpha P_{s}}_{a_{1}} s \exp \left(\frac{\sqrt{2} 2 \sigma_{h_{1}} x_{n}+2 \mu_{h_{1}}}{\xi}\right)]
$$

$$
\Psi_{\mathcal{Y}+a_{3}}(s) \triangleq \exp (\underbrace{-2 \eta \alpha d_{1}^{m} \sigma_{r}^{2}}_{a_{3}} s) \sum_{n=1}^{N} \frac{w_{n}}{\sqrt{\pi}} \exp [\underbrace{-(1-\alpha) d_{1}^{m} d_{2}^{m} \sigma_{d}^{2}}_{a_{2}} s \exp \left(-\frac{\sqrt{2} 2 \sigma_{h_{2}} x_{n}+2 \mu_{h_{2}}}{\xi}\right)]
$$

$$
P_{\text {out }}=\int_{0}^{\frac{v a_{3}}{a_{1}}} f_{X}(z) \underbrace{\operatorname{Pr}\left(Y>\frac{v a_{2}}{a_{1} z-v a_{3}}\right)}_{=1} \mathrm{~d} z+\int_{\frac{v a_{3}}{a_{1}}}^{\infty} f_{X}(z) \operatorname{Pr}\left(Y \leq \frac{v a_{2}}{a_{1} z-v a_{3}}\right) \mathrm{d} z,
$$

$$
P_{\text {out }}=1-\frac{\xi}{4 \sqrt{8 \pi \sigma_{h_{1}}^{2}}} \int_{\frac{v a_{3}}{a_{1}}}^{\infty} \frac{1}{z}\left(1+\operatorname{erf}\left[\frac{\xi \ln (\Gamma)-2 \mu_{h_{2}}}{2 \sqrt{2} \sigma_{h_{2}}}\right]\right) \exp \left[-\frac{\left(\xi \ln (z)-\left(2 \mu_{h_{1}}+\xi \ln \left(a_{1}\right)\right)\right)^{2}}{8 \sigma_{h_{1}}^{2}}\right] \mathrm{d} z
$$

The probability in the first integral of (23) is equal to 1 , because $Y$ is always positive which will always be greater than the negative value of $\frac{v a_{2}}{a_{1} z-v a_{3}}$ when $0<z<\frac{v a_{3}}{a_{1}}$ ), whereas the one in the second integral represents the cumulative distribution functions (CDF) of the RV, $Y$. Hence, the outage probability can now be simplified to

$$
P_{\text {out }}=\int_{0}^{\frac{v a_{3}}{a_{1}}} f_{X}(z) \mathrm{d} z+\int_{\frac{v a_{3}}{a_{1}}}^{\infty} f_{X}(z) F_{Y}(z) \mathrm{d} z .
$$

where $F_{Y}($.$) denotes the \mathrm{CDF}$, and is given in this case by

$$
F_{Y}(\Gamma)=\frac{1}{2}\left(1+\operatorname{erf}\left[\frac{\xi \ln (\Gamma)-2 \mu_{h_{2}}}{2 \sqrt{2} \sigma_{h_{2}}}\right]\right),
$$

where erf (.) is the error function and $\Gamma=\frac{v a_{2}}{z-v a_{3}}$. Substituting (24) and (26) into (25) yields the TSR system's outage probability given by (27), shown at the top of the next page.

\section{Power Splitting Relaying}

This protocol divides the block time, $T$, into two equal periods, i.e. $T / 2$, for source-to-relay and relay-to-destination transmissions as illustrated in Fig. 3a. During the first half, the relay allocates a portion of the received signal power to the energy-harvester, $\rho P$, whereas the remaining power, $(1-\rho) P$, is used for information transmission as presented in Fig. 3b. Hence, the received signal at the input of the energy-harvester can be expressed as

$$
\sqrt{\rho} y_{r}(t)=\frac{1}{\sqrt{d_{1}^{m}}} \sqrt{\rho P_{s}} h_{1} s(t)+\sqrt{\rho} n_{a}(t) .
$$

The resultant harvested energy can now be simply written as

$$
E_{H}=\frac{\eta \rho T}{2}\left(\frac{P_{s}}{d_{1}^{m}} h_{1}^{2}+\sigma_{a}^{2}\right) .
$$

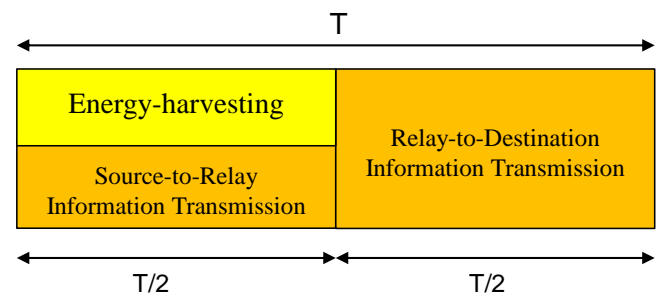

(a) Time frame structure in the PSR protocol.

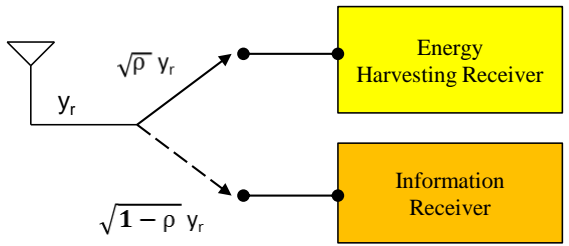

(b) Block diagram of the PSR-based relaying system.

Figure 3: PSR protocol for EH and information signal processing at the relay.

Using this harvested energy, the relay amplifies and forwards the source signal to the destination node. Hence, the transmitted signal at the relay can be given by

$$
r(t)=\frac{1}{\sqrt{d_{1}^{m}}} \sqrt{(1-\rho) P_{s} P_{r}} G h_{1} s(t)+\sqrt{P_{r}} G n_{r}(t),
$$

where $n_{r}(t)=\sqrt{1-\rho} n_{a}(t)+n_{c}(t)$ and $G$ is given for this system as $G=\frac{1}{\sqrt{\frac{(1-\rho) P_{s}}{d_{1}^{n}} h_{1}^{2}+\sigma_{r}^{2}}}$. From (30), the received signal at the destination can be written as

$$
y_{D}(t)=\frac{1}{\sqrt{d_{1}^{m} d_{2}^{m}}} \sqrt{(1-\rho) P_{s} P_{r}} G h_{1} h_{2} s(t)
$$




$$
+\frac{1}{\sqrt{d_{2}^{m}}} \sqrt{P_{r}} G h_{2} n_{r}(t)+n_{d}(t) .
$$

The transmitted power of the relay as a function of the harvested energy is given by $P_{r}=\frac{2 E_{H}}{T / 2}$, which can also be expressed, using (29), as

$$
P_{r}=\eta \rho\left(\frac{P_{s}}{d_{1}^{m}} h_{1}^{2}+\sigma_{a}^{2}\right) .
$$

Substituting (32) into (31), and with some algebraic manipulation, the SNR at the destination node in the PTS based system can expressed as

$$
\gamma_{D}=\frac{\eta \rho(1-\rho) P_{s} h_{1}^{2} h_{2}^{2}}{\eta \rho d_{1}^{m} \sigma_{c}^{2} h_{2}^{2}+\eta \rho(1-\rho) d_{1}^{m} \sigma_{a}^{2} h_{2}^{2}+(1-\rho) d_{1}^{m} d_{2}^{m} \sigma_{d}^{2}}
$$

\section{A. Ergodic Capacity for the PSR Protocol}

Below, we derive an expression for the ergodic capacity for the PSR based system over the log-normal fading channel. Firstly, let

$$
\begin{aligned}
& b_{1}=\eta \rho(1-\rho) P_{s}, \\
& b_{2}=(1-\rho) d_{1}^{m} d_{2}^{m} \sigma_{d}^{2}, \\
& b_{3}=\eta \rho d_{1}^{m} \sigma_{c}^{2}, \\
& b_{4}=\eta \rho(1-\rho) d_{1}^{m} \sigma_{a}^{2}, \\
& \mathcal{Q}=b_{1} X, \text { and } \\
& \mathcal{R}=b_{2} \bar{Y} .
\end{aligned}
$$

Using these definitions, we can straightforwardly rewrite (33) as

$$
\gamma_{D}=\frac{\mathcal{Q}}{\mathcal{R}+b_{3}+b_{4}} .
$$

From (5) and (35), the ergodic capacity for this system can also be given as

$$
\mathbb{E}\left[C_{D}\right]=\frac{1}{2} \mathbb{E}\left[\log _{2}\left(1+\frac{\mathcal{Q}}{\mathcal{R}+b_{3}+b_{4}}\right)\right] .
$$

Now, using (16), we can rewrite the ergodic capacity of the PSR system (36) in the following form

$$
\mathbb{E}\left[C_{D}\right]=\frac{1}{2 \ln (2)} \int_{0}^{\infty} \frac{1}{s}\left(1-\Psi_{\mathcal{Q}}(s)\right) \Psi_{\mathcal{R}+b_{3}+b_{4}}(s) \mathrm{d} s,
$$

where $\Psi_{\mathcal{Q}}(z)$ and $\Psi_{\mathcal{R}+b_{3}+b_{4}}(z)$ are the MGFs of the RVs $\mathcal{Q}$ and $\mathcal{R}+b_{3}+b_{4}$, and are given, respectively, as $\Psi_{\mathcal{Q}}(s)$ $=\Psi_{X}\left(b_{1} s\right)$ and $\Psi_{\mathcal{R}+b_{3}+b_{4}}(s)=\Psi_{\mathcal{R}}(s) \exp \left(-b_{3} s\right)$ $\exp \left(-b_{4} s\right)$, where $\Psi_{\mathcal{R}}(s)=\Psi_{\bar{Y}}\left(b_{2} s\right)$. Now, since the channel is log-normally distributed, we can express $\Psi_{\mathcal{Q}}$ and $\Psi_{\mathcal{R}+b_{3}+b_{4}}$ as in (38) and (39), respectively, shown at the top of the next page. Finally, substituting (38) and (39) into (37) yields the destination ergodic capacity of the PSR EH system over log-normal fading channels.

\section{B. Ergodic Outage Probability for the PSR Protocol}

Following the same procedure as in the previous section, it is straightforward to show that the outage probability of the PSR protocol over the log-normal fading channel can be expressed as

$$
\begin{aligned}
P_{\text {out }} & =1-\frac{\xi}{\sqrt{8 \pi \sigma_{h_{1}}^{2}}} \int_{\frac{v\left(b_{3}+b_{4}\right)}{b_{1}}}^{\infty}\left(\frac{1}{z}\right. \\
& \times\left(1-\frac{1}{2}\left(1+\operatorname{erf}\left[\frac{\xi \ln (\Lambda)-2 \mu_{h_{2}}}{2 \sqrt{2} \sigma_{h_{2}}}\right]\right)\right) \\
& \left.\times \exp \left[-\frac{\left(\xi \ln (z)-2 \mu_{h_{1}}-\xi \ln \left(b_{1}\right)\right)^{2}}{8 \sigma_{h_{1}}^{2}}\right]\right) \mathrm{d} z
\end{aligned}
$$

where

$$
\Lambda=\frac{v b_{2}}{b_{1} z-v b_{2}-v b_{4}} .
$$

\section{Numerical Results}

In this section, we present numerical examples for the ergodic capacity and the ergodic outage probability expressions derived above. Monte Carlo simulations are also provided throughout to verify our analysis. The system parameters adopted here, unless clearly stated otherwise, are as follows: $m=2.7, G=1, \sigma_{r}^{2}=\sigma_{d}^{2}=0.01, \sigma_{a}^{2}=\sigma_{c}^{2}=\sigma_{r}^{2} / 2$, $\mu_{h_{1}}=\mu_{h_{2}}=2$, and $\sigma_{h_{1}}^{2}=\sigma_{h_{2}}^{2}=2$.

\section{A. Ergodic Capacity}

In this section, we investigate the impact of $\alpha, \rho, \sigma_{h_{1}}^{2}$ and $\sigma_{h_{2}}^{2}$ on the ergodic capacity of the TSR and PSR based systems. System parameters considered here are $P_{s}=1, d_{1}=d_{2}=3$ and $\eta=1$. Figs. $4 \mathrm{a}$ and $4 \mathrm{~b}$ illustrate some analytical and simulated results for the ergodic capacities as a function of $\alpha$ and $\rho$, respectively, for various variances of the log-normal channel. The analytical results for the TSR and PSR systems are obtained from (17) and (37), respectively. It is interesting to observe that as the channel variance increases, both $\alpha$ and $\rho$ decrease which implies that less EH is now required to maintain the same ergodic capacity. It is also evident that for both systems there always exists an optimal $\mathrm{EH}$ time, $\alpha^{*}$, and an optimal power splitting factor, $\rho^{*}$, that maximize the system performance. This is because when $\alpha$ is too small in the TSRbased system, there is no sufficient time for $\mathrm{EH}$ and hence only a small amount of energy is harvested which of course will result in poor capacity. At the other extreme, when $\alpha$ is too large, too much energy is harvested unnecessarily at the expense of information transmission time which, consequently, leads to poor capacity. Similarly, this justification applies to $\rho$ in the PSR system. It is clearly noted therefore that the performance of these protocols are greatly dependent on the selection of $\alpha$ and $\rho$. This phenomena is studied in more detail below. 


$$
\Psi_{\mathcal{Q}}(s) \triangleq \sum_{n=1}^{N} \frac{w_{n}}{\sqrt{\pi}} \exp \left[-\eta \rho(1-\rho) P_{s} s \exp \left(\frac{\sqrt{2} 2 \sigma_{h_{1}} x_{n}+2 \mu_{h_{1}}}{\xi}\right)\right]
$$

$$
\Psi_{\mathcal{R}+b_{3}+b_{4}}(s) \triangleq \exp \left(\eta \rho d_{1}^{m}\left(\rho \sigma_{a}^{2}-\sigma_{c}^{2}-\sigma_{a}^{2}\right) s\right) \sum_{n=1}^{N} \frac{w_{n}}{\sqrt{\pi}} \exp \left[(\rho-1) d_{1}^{m} d_{2}^{m} \sigma_{d}^{2} s \exp \left(-\frac{2 \sqrt{2} \sigma_{h_{2}} x_{n}+2 \mu_{h_{2}}}{\xi}\right)\right]
$$

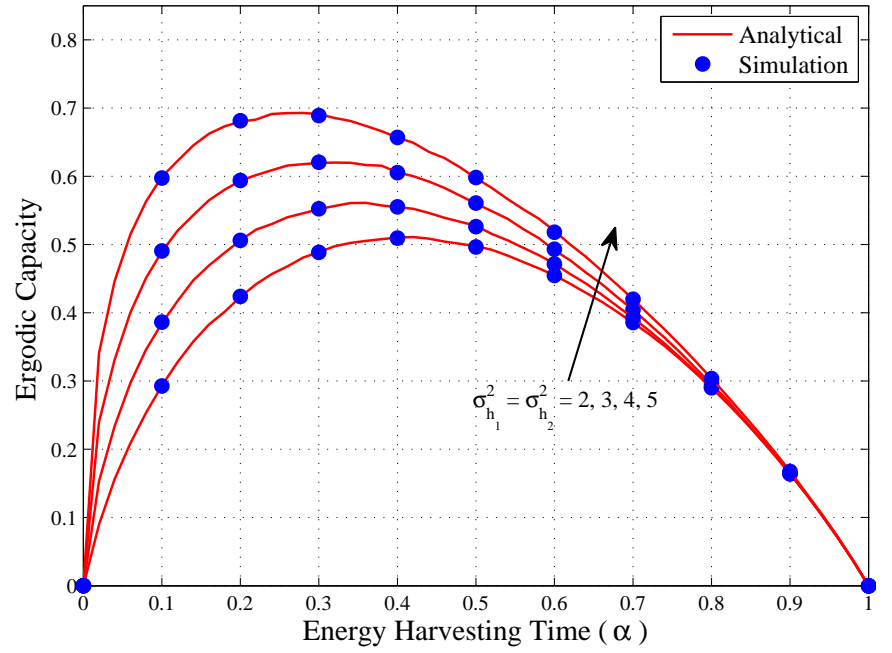

(a) TSR

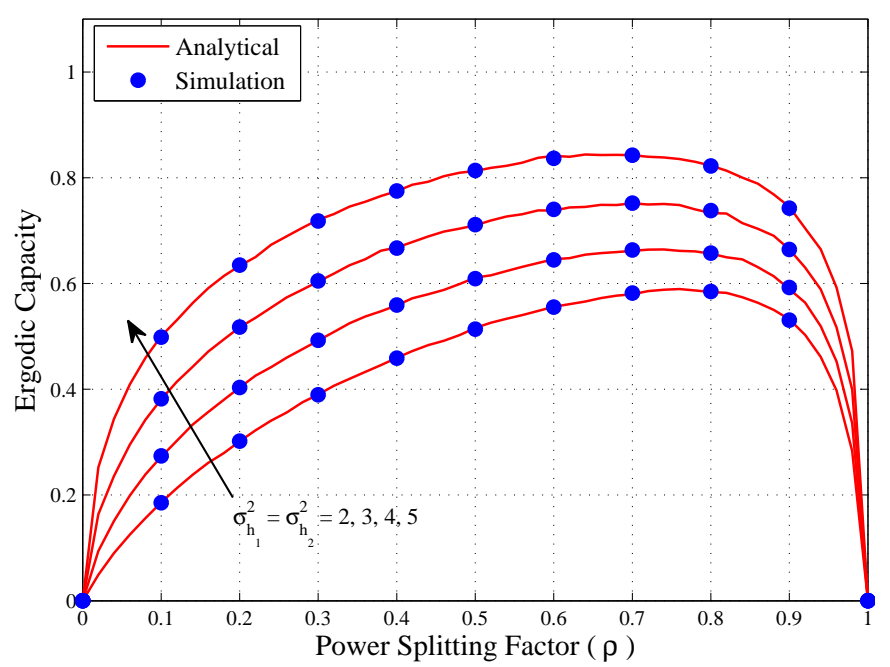

(b) PSR

Figure 4: Ergodic capacity versus the EH time and power splitting factor for the TSR and PSR protocols with different variances of the log-normal channel.

\section{B. Optimized Systems}

We now evaluate the performance of the optimized TSR and PSR systems. To begin with, we plot in Fig. 5 the optimal $\alpha$ and $\rho$ with different values of $P_{s}$ and $\eta=1$, when the relay is moved from the source $(0,0)$ towards the destination $(0,10)$ such that $d_{1}=10-d_{2}$. The analytical results of these parameters can be easily evaluated from (17) and (37) by solving the following

$$
\frac{\partial}{\partial \alpha / \rho}\left\{\mathbb{E}\left[C_{D}\right]\right\}=0
$$

Although it is not easy to express the solution of these equations in closed-form, numerical solutions do not pose any computational difficulties and can be determined with the help of some built-in functions in software tools such as the FindMaximum function in Mathematica. It can be seen from Fig. 5 that the relay location greatly affects the optimized EH parameters. The other observation one can see is that at a given location, increasing the source power will reduce both $\alpha^{*}$ and $\rho^{*}$ but more so for the former. For the TSR-based system, the longest harvesting time occurs when the relay is positioned around half-way between the source and the destination. The final remark on these results is that in both the TSR and PSR protocols, $\alpha^{*}$ and $\rho^{*}$ approach zero as the relay becomes very close to the source or relay which is intuitive.

Fig. 6 depicts the maximum achievable ergodic capacity corresponding to $\alpha^{*}$ and $\rho^{*}$ shown in Fig. 5. It is evident that the optimized PSR system always outperforms the optimized
TSR irrespective of the relay location. In addition, the lowest ergodic capacity for the two systems is noticed when the relay location is around midway. This is because in this region, $\mathrm{EH}$ is at its peak, as illustrated in Fig. 5, which considerably affects information transmission time and hence the overall ergodic capacity.

\section{Ergodic Outage Probability}

The ergodic outage probability of the systems under consideration is examined in this section. Fig. 7 presents some numerical examples of this probability for the optimized TSR and PSR schemes with two system settings: $\left\{\eta=0.4, P_{s}=1\right\}$ and $\left\{\eta=1, P_{s}=5\right\}$ when $\sigma_{h_{1}}^{2}=\sigma_{h_{2}}^{2}=3$ and $d_{1}=d_{2}=1$. The analytical results of the TSR and PSR systems are obtained from (27) and (40), respectively, when using $\alpha^{*}$ and $\rho^{*}$. For the first system setting we use $\alpha^{*}=0.154$ and $\rho^{*}=0.581$ and for the second, we use $\alpha^{*}=0.101$ and $\rho^{*}=0.491$. It is seen from these results that the PSR system always has lower ergodic outage probability and that increasing $\eta$ and/or $P_{s}$ will considerably boost the performance for the two systems under investigation.

\section{CONCLUSION}

In this paper we analyzed the performance of different EH protocols in cooperative communication systems over lognormal fading channels. Two EH protocols were considered namely, TSR and PSR. Accurate analytical expressions for the ergodic capacity and ergodic outage probability for the two 


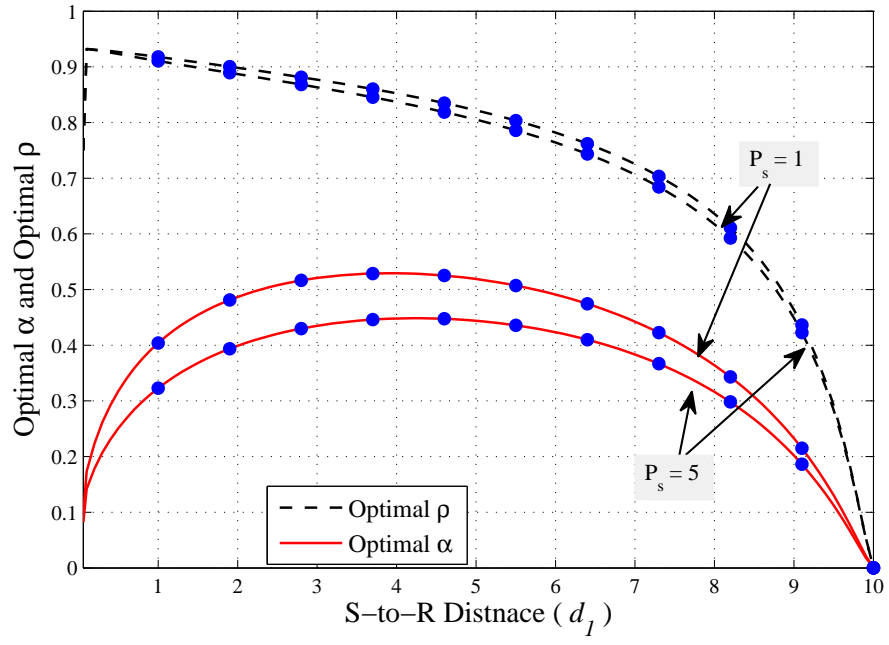

Figure 5: Optimal EH time and optimal power slipping factor versus the source-to-relay distance $\left(d_{1}=10-d_{2}\right)$ when $P_{s}=1$ and 5 . The solid and dashed lines represent the analytical results whereas the blue circles indicate the simulated results.

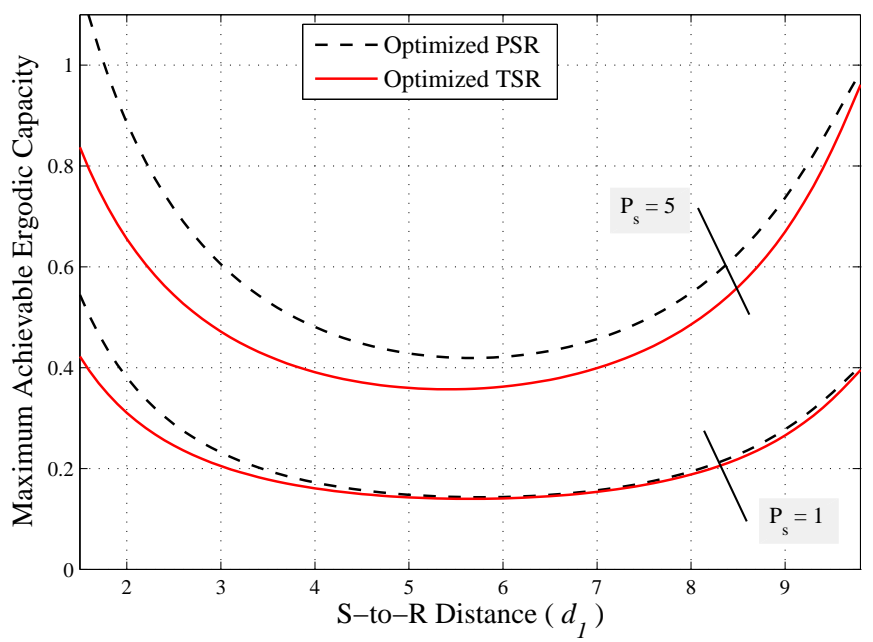

Figure 6: The maximum achievable ergodic capacity for the TSR and PSR schemes over the log-normal fading channel versus the S-to-R distance $\left(d_{1}=10-d_{2}\right)$ when $P_{s}=1$ and 5 .

systems were derived and validated with Monte Carlo simulations. The results demonstrated that as the channel variance increases, the EH based systems will provide better ergodic capacity. Also, a good selection of the EH time in the TSR protocol, and the power splitting factor in the PTS protocol, was found to be the key to achieving the best performance.

\section{REFERENCES}

[1] X. Zhou, R. Zhang, and C. K. Ho, "Wireless information and power transfer: Architecture design and rate-energy tradeoff," IEEE Trans. Commun., vol. 61, pp. 4754-4767, Nov. 2013.

[2] K. Huang and E. Larsson, "Simultaneous information and power transfer for broadband wireless systems," IEEE Trans. Signal Process., vol. 61, pp. 5972-5986, Dec. 2013.

[3] I. Krikidis, S. Timotheou, and S. Sasaki, "RF energy transfer for cooperative networks: Data relaying or energy harvesting?" IEEE Commun. Lett., vol. 16, pp. 1772-1775, Nov. 2012.

[4] A. Nasir, X. Zhou, S. Durrani, and R. Kennedy, "Relaying protocols for wireless energy harvesting and information processing," IEEE Trans. Wireless Commun., vol. 12, pp. 3622-3636, Jul. 2013.

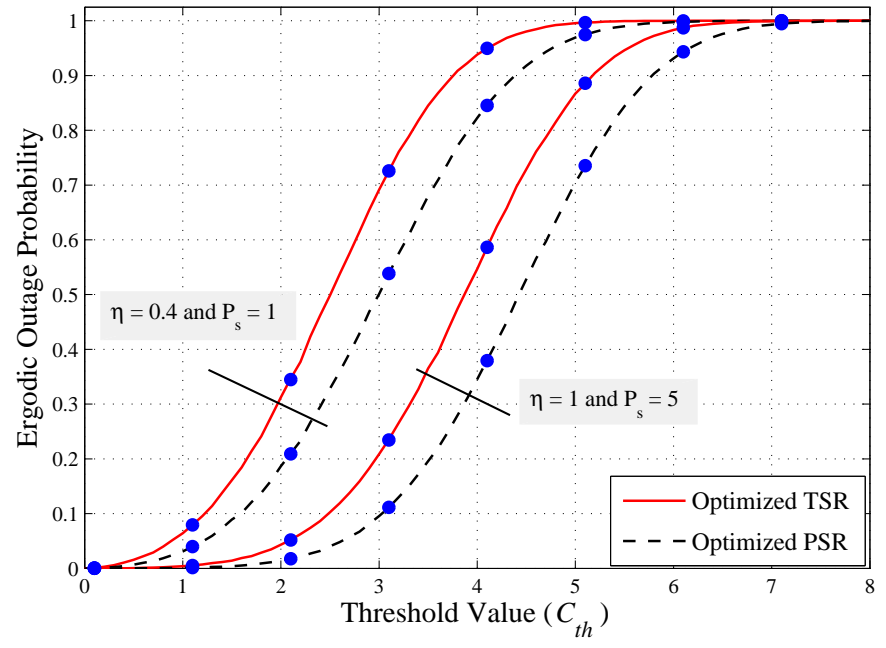

Figure 7: Ergodic outage probability for the two EH protocols over a lognormal fading channel with different system settings. The solid and dashed lines represent the analytical results whereas the blue circles represent Monte Carlo simulation results.

[5] Z. Chen, B. Xia, and H. Liu, "Wireless information and power transfer in two-way amplify-and-forward relaying channels," in Proc. IEEE Global Conf. Signal and Inf. Process. (GlobalSIP), pp. 168-172, Atlanta, GA, Dec. 2014.

[6] I. Krikidis, "Simultaneous information and energy transfer in large-scale networks with/without relaying," IEEE Trans. Commun., vol. 62, pp. 900912, Mar. 2014.

[7] Z. Ding, S. Perlaza, I. Esnaola, and H. Poor, "Power allocation strategies in energy harvesting wireless cooperative networks," IEEE Trans. Wireless Commun., vol. 13, pp. 846-860, Feb. 2014.

[8] D. Michalopoulos, H. Suraweera, and R. Schober, "Relay selection for simultaneous information transmission and wireless energy transfer: A tradeoff perspective," IEEE Trans. J. Sel. Areas in Commun., vol. 33, pp. 1578-1594, Aug. 2015.

[9] S. Seidel, K. Takamizawa, and T. Rappaport, "Application of second-order statistics for an indoor radio channel model," in Proc. Veh. Technol. Conf. (VTC), pp. 888-892, San Francisco, CA, May 1989.

[10] D. Cassioli, M. Win, and A. Molisch, "The ultra-wide bandwidth indoor channel: from statistical model to simulations," IEEE J. Sel. Areas in Commun., vol. 20, pp. 1247-1257, Aug. 2002.

[11] M. K. Simon and M.-S. Alouini, Digital Communication Over Fading Channels. 2005.

[12] N. Mehta, J. Wu, A. Molisch, and J. Zhang, "Approximating a sum of random variables with a lognormal," IEEE Trans. Wireless Commun., vol. 6, pp. 2690-2699, Jul. 2007.

[13] M. Abramowitz and I. A. Stegun, Handbook of Mathematical Functions with Formulas, Graphs, and Mathematical Tables. 1972.

[14] S. Zhang and V. Lau, "Multi-relay selection design and analysis for multistream cooperative communications," IEEE Trans. Wireless Commun., vol. 10, pp. 1082-1089, Apr. 2011.

[15] M. Xia and S. Aissa, "Cooperative af relaying in spectrum-sharing systems: Outage probability analysis under co-channel interferences and relay selection," IEEE Trans. Commun., vol. 60, pp. 3252-3262, Nov. 2012.

[16] M. Di Renzo and W. Lu, "End-to-end error probability and diversity analysis of AF-based dual-hop cooperative relaying in a Poisson field of interferers at the destination," IEEE Trans. Wireless Commun., vol. 14, pp. 15-32, Jan. 2015.

[17] H.-M. Wang, M. Luo, X.-G. Xia, and Q. Yin, "Joint cooperative beamforming and jamming to secure AF relay systems with individual power constraint and no eavesdropper's CSI,' IEEE Signal Process. Lett., vol. 20, pp. 39-42, Jan. 2013.

[18] K. Hamdi, "A useful lemma for capacity analysis of fading interference channels," IEEE Trans. Commun., vol. 58, pp. 411-416, Feb. 2010. 